\title{
1-benzyl-1,4-diazepane reduces the efflux of resistance-nodulation-cell division pumps in Escherichia coli
}

\author{
Enrico Casalone*,1 (D), Tiziano Vignolini ${ }^{2}$, Laura Braconi ${ }^{3}$, Lucia Gardini ${ }^{2,4}$, Marco \\ Capitanio $^{2,5}$, Francesco S Pavone ${ }^{2,4,5}$, Silvia Dei $^{3}$ \& Elisabetta Teodori ${ }^{3}$ \\ ${ }^{1}$ Department of Biology, University of Florence, Via Madonna del Piano 6, 50019 Sesto Fiorentino, Italy \\ ${ }^{2}$ LENS - European Laboratory for Non-linear Spectroscopy, Via Nello Carrara 1, 50019 Sesto Fiorentino, Italy \\ ${ }^{3}$ Department of Neuroscience, Psychology, Drug Research \& Child Health (NEUROFARBA), Via U. Schiff, 6 - 50019 Sesto \\ Fiorentino, Italy \\ ${ }^{4}$ National Institute of Optics-National Research Council, Largo Fermi 6, 50125 Florence, Italy \\ ${ }^{5}$ Department of Physics \& Astronomy, University of Florence, Via Sansone 1, 50019 Sesto Fiorentino, Italy \\ *Author for correspondence: enrico.casalone@unifi.it
}

Aim: To investigate the action mechanism of 1-benzyl-1,4-diazepane (1-BD) as efflux pump inhibitor (EPI) in Escherichia coli mutants: $\triangle a c r A B$ or overexpressing $A c r A B$ and AcrEF efflux pumps. Materials \& methods: Effect of 1-BD on: antibiotic potentiation, by microdilution method; membrane functionality, by fluorimetric assays; ethidium bromide accumulation, by fluorometric real-time efflux assay; AcrB expression, by quantitative photoactivated localization microscopy. Results: 1-BD decreases the minimal inhibitory concentration of levofloxacin and other antibiotics and increase ethidium bromide accumulation in $E$. coli overexpressing efflux pumps but not in the $\triangle a c r A B$ strain. 1-BD increases membranes permeability, without sensibly affecting inner membrane polarity and decreases $a c r A B$ transcription. Conclusion: 1-BD acts as an EPI in E. coli with a mixed mechanism, different from that of major reference EPIs.

\section{Graphical abstract:}
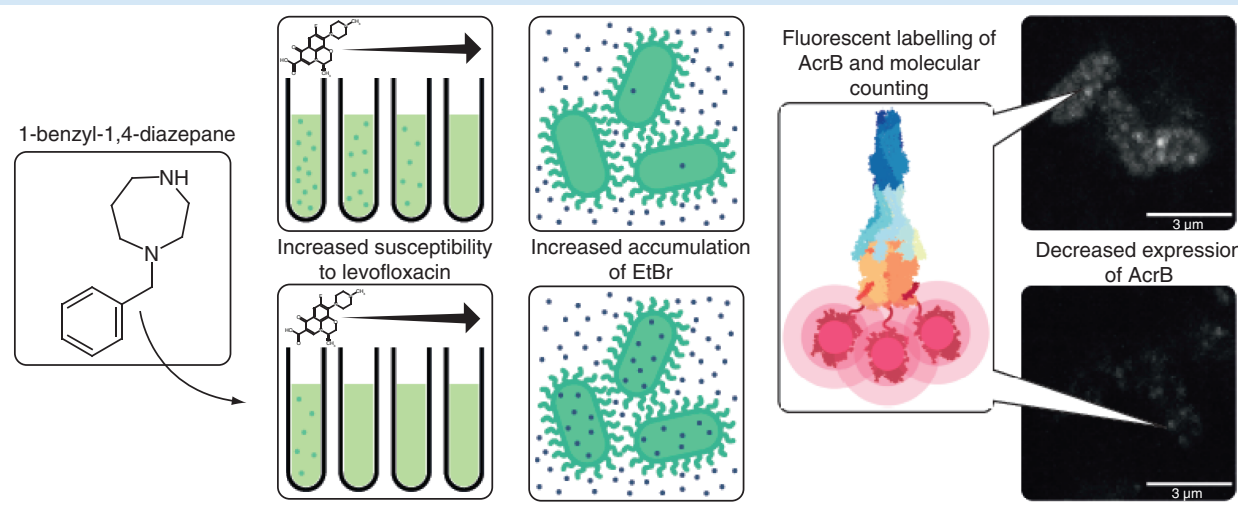

First draft submitted: 25 October 2019; Accepted for publication: 10 July 2020; Published online: 25 August 2020

Keywords: AcrAB expression $\bullet$ E. coli $\bullet$ efflux-pump inhibitor $\bullet$ EPI $\bullet$ levofloxacin potentiation $\bullet$ MDR $\bullet$ membrane permeability $\bullet$ multidrug resistance

The large and often incorrect use of antibiotics has exerted selective pressure on susceptible bacteria, favoring resistant strains over time. One of the most successful strategies the bacteria use to adapt to chemically unrelated antimicrobial agents relies on the activation of ubiquitous efflux pumps (EPs) to extrude the drug [1-3]. In Gramnegative bacteria (GNB), the increased expression of EP genes acts synergistically with the outer membrane $(\mathrm{OM})$, 

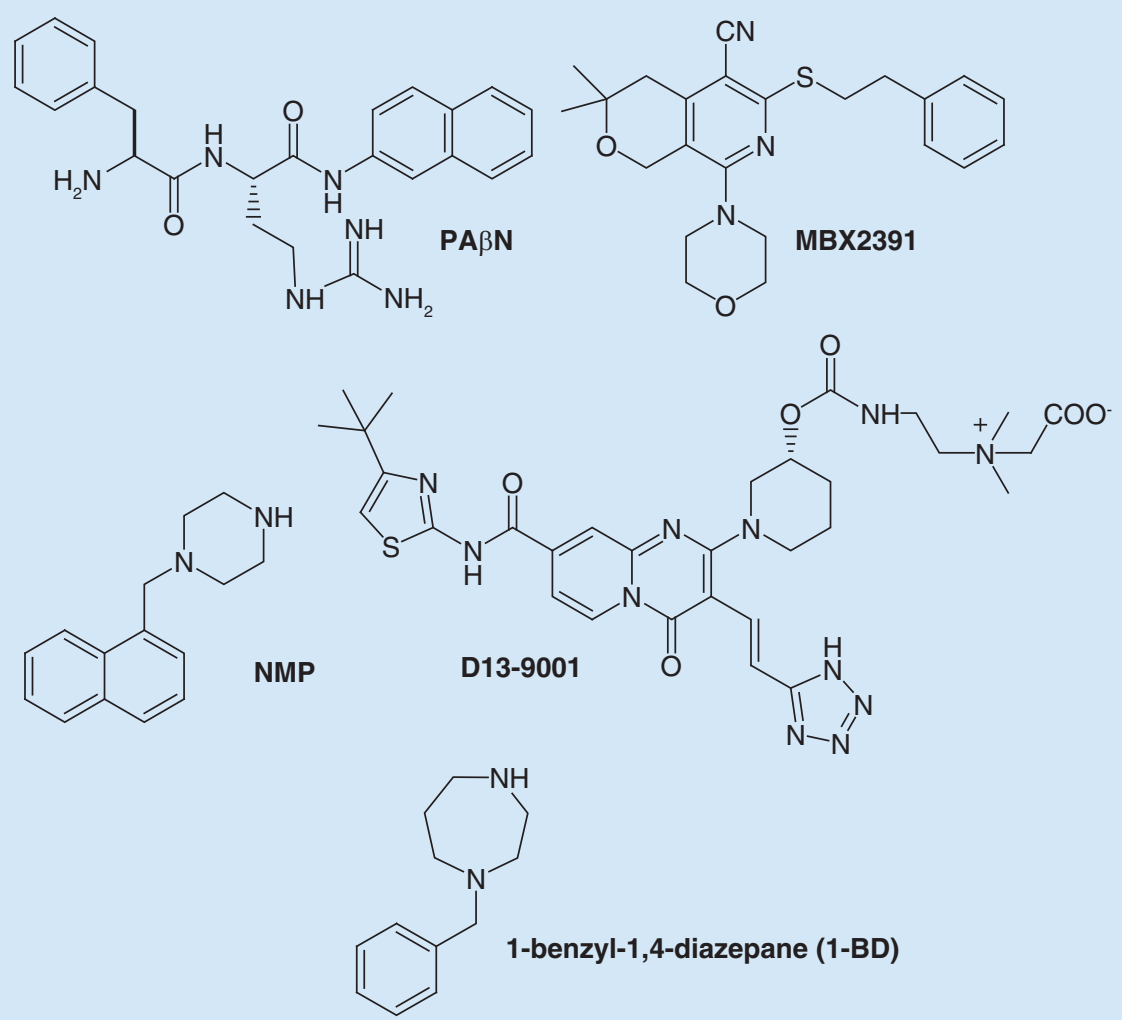

1-benzyl-1,4-diazepane (1-BD)

Figure 1. Structures of the main inhibitors of Escherichia coli resistance-nodulation-cell division efflux pumps and of 1-benzyl-1,4-diazepane.

resulting in a multidrug-resistant (MDR) phenotype $[1,4]$. AcrAB, belonging to the so-called resistance-nodulationcell division (RND) family of bacterial EPs [1], is the main representative of the MDR-EPs in Escherichia coli [1,5]. $\mathrm{Acr} A B$ is predominant in GNB and associated with MDR in most clinically significant strains [1]. AcrAB-TolC is a tripartite complex (AcrA, AcrB and TolC), spanning across the inner membrane (IM) and OM of GNB [6,7] and can drive the extrusion of antibiotics directly from the cytoplasm into the external medium. RND pumps use proton motive force (PMF) as a source of energy and the IM protein AcrB catalyzes drug/ $\mathrm{H}^{+}$antiport, in addition to being responsible for drug selectivity [7]. Deletions in $a c r A B$ or tolC genes resulted in increased susceptibility to a wide range of antibiotics and other toxic compounds [8]. Other RND EPs, like AcrEF and AcrAD, are also present in E. coli [9].

In EP-dependent MDR bacteria, the resistant phenotype may be reversed by using efflux pump inhibitors (EPIs), which act by increasing the intracellular concentration of the antibiotics [10]. The utilization of EPIs as adjuvants in antibiotic therapies is potentially of great interest in the battle against MDR pathogens [10,11]. Several natural and synthetic compounds are capable of inhibiting RND EPs in E. coli and other GNB [12]. Phenylalanine-arginine $\beta$-naphthylamide $(\mathrm{PA} \beta \mathrm{N})$, a synthetic dipeptide amine, MBX2391, a pyranopyrimidine, 1-(1-naphthylmethyl)piperazine (NMP), a piperazine derivative and the pyridopyrimidine D13-9001, are among the best characterized EPIs (Figure 1). Existing non-antibacterial drugs also demonstrated inhibitory activity by a more or less direct interaction with AcrAB-TolC [12]. However, problems like toxicity to humans, low water solubility and high affinity to serum albumin do not permit to any EPI to reach a clinical stage.

Expression of AcrAB in Enterobacteriaceae is subject to multiple levels of regulation [13]. In E. coli the expression is primarily controlled by the multiple antibiotic resistance (mar) operon by the global transcriptional activator MarA. MarA induces $a c r A B$ and tolC transcription and, increasing drug efflux, determined an MDR phenotype. MarR, another product of Mar operon, represses marA transcription. MarR has a strong affinity for aromatic molecules and MarA repression can be removed by phenolic compounds such as sodium salicylate [13] or diazepam, 


\begin{tabular}{|c|c|c|c|c|c|}
\hline \multirow[t]{2}{*}{ Strain } & \multicolumn{4}{|c|}{ Genotype } & \multirow[t]{2}{*}{ Pump-related phenotype } \\
\hline & gyrA & mar & acrR or $\operatorname{acr} A B$ & acrS or acrEF & \\
\hline DC14PS & wt & wt & Inactivated ${ }^{\dagger}$ & wt & Not expressing $A c r A B$ \\
\hline 1-DC14PS & S83L & wt & Inactivated & wt & Not expressing AcrAB \\
\hline 2-DC14PS & S83L & wt & Inactivated & IS2 inserted & $\begin{array}{l}\text { Overexpressing AcrEF not } \\
\text { expressing } A c r A B\end{array}$ \\
\hline 3-AG100MKX & S83L & Inactivated & IS186 inserted & wt & Overexpressing $A c r A B$ \\
\hline TV001 & wt & wt & SacrB::PAmCherry & wt & $\begin{array}{l}\text { Expressing } \\
\text { AcrAB-PamCherry }\end{array}$ \\
\hline
\end{tabular}

$\dagger^{\dagger}$ Gene inactivation in all strains was always obtained by insertion of $a \mathrm{Kan}^{R}$ cassette.

‡single mutation.

wt: Wild-type.

a benzodiazepine sedative drug [14,15] in E. coli, resulting in a MDR phenotype. The local repressor AcrR acts as a modulator to prevent the overexpression of $\operatorname{acr} A B$ [13].

During a screening of a number of piperazines, aimed to the identification of putative EPIs, we found that the homolog 1-benzyl-1,4-diazepane (1-BD), also called 1-benzyl-1,4-homopiperazine (Figure 1), had some effect in reversing levofloxacin (LEV) resistance in E. coli mutants overexpressing RND EPs. The aim of this paper is to characterize the mechanism of action of 1-BD in the inhibition of the main RND EPs in E. coli.

\section{Materials \& methods}

\section{Chemicals}

Levofloxacin, chloramphenicol (CHL), tetracycline (TET), rifampicin (RIF) and ethidium bromide (EtBr) were from Sigma-Aldrich Inc (MO, USA). NMP was from FluoroChem (Hadfield, UK), it was used as reference EPI [16]. 1-BD was a high-purity commercial compound from Sigma-Aldrich (Milan, Italy). All these molecules were dissolved in water. 1-BD showed a good water solubility and was dissolved in this solvent at a concentration of $13.3 \mathrm{mg} / \mathrm{ml}(0.07 \mathrm{M}$ approximately).

\section{Bacterial strains \& growth conditions}

All the strains used in this study are listed in Table 1. The AcrAB pump-deficient and the AcrAB and AcrEF overexpressing E. coli strains are derivatives of the AG100 E. coli strain [17]. All these strains carried a $\operatorname{Kan}^{\mathrm{R}}$ cassette, which allowed us to select them on Luria Bertani (LB) agar in the presence of $100 \mu \mathrm{g} / \mathrm{ml}$ of kanamycin, before using the cells in subsequent assays. E. coli strains were grown in LB at $37^{\circ} \mathrm{C}$. The E. coli strain TV001 used for the photoactivated localization microscopy (PALM) experiments is a derivative of the E. coli K12 strain BW25113 [18]. TV001 ( $2 a c r B:: P A m C h e r r y)$ was engineered by creating a fusion between the chromosomal acrB gene of BW25113 and a sequence encoding for PAmCherry, a photoactivatable fluorescent protein [19], through CRISPR/Cas9-assisted recombineering [20]. As such, all instances of AcrB expressed by TV001 are fused with a PAmCherry moiety [21]. Both BW25113 and TV001 were grown in LB with no added antibiotic.

\section{Bacterial susceptibility testing}

Susceptibility tests were performed by a twofold standard microdilution method following the Clinical and Laboratory Standards Institute recommendations [22]. Overnight cultures of E. coli were suitably diluted and inoculated $(50 \mu \mathrm{l})$ in a 96 -well Microtiter ${ }^{\circledR}$ plates to obtain approximately $5 \times 10^{5} \mathrm{CFU} / \mathrm{ml}$ in a final volume of $150 \mu \mathrm{l} /$ well in the presence and absence (control) of different compounds. Minimum inhibitory concentration (MIC) values were detected in an Infinite M200 PRO Tecan microplate reader (Tecan ${ }^{\circledR}$ France, SA-Lyon, France) by reading optical density (OD) at wavelength $600 \mathrm{~nm}\left(\mathrm{OD}_{600 \mathrm{~nm}}\right)$ after $24 \mathrm{~h}$ incubation. The adjuvant-like effect of subtoxic concentrations ( $\leq 1 / 4 \mathrm{MIC})$ of 1-BD with LEV, CHL, TET and RIF were evaluated by three independent experiments carried out in duplicate. The results were expressed by the activity gain parameter $(\mathrm{A}): \mathrm{A}=\mathrm{MIC}_{\mathrm{ant}} / \mathrm{MIC}_{\mathrm{ant}+1-\mathrm{BD}}$, where $\mathrm{MIC}_{\text {ant }}$ is the MIC value of the antibiotic alone and $\mathrm{MIC}_{\mathrm{ant}+1-\mathrm{BD}}$ the MIC of antibiotic in the presence of 1-BD; the higher the A value, the greater the adjuvant effect of 1-BD toward the antibiotic. Checkerboard assay was performed in duplicate, essentially as described previously [23], on the 1-DC14PS, 2-DC14PS and 3-AG100MKX strains by assembling a 2D array of serial dilutions of $1-\mathrm{BD}(3.2-0.05 \mathrm{mg} / \mathrm{ml})$ and LEV $(16-0.125 \mu \mathrm{g} / \mathrm{ml})$ in a 96-well Microtiter plate. The interaction between 1-BD and LEV was evaluated by the fractional inhibitory 
concentration $(\mathrm{FIC})$ index $(\mathrm{FICi}): \mathrm{FICi}=\mathrm{FIC}_{\mathrm{LEV}}+\mathrm{FIC}_{1-\mathrm{BD}}$, where $\mathrm{FIC}_{\mathrm{LEV}}=\mathrm{MIC}_{\mathrm{LEV}}+1-\mathrm{BD} / \mathrm{MIC} \mathrm{LEV}$ and $\mathrm{FIC}_{1-\mathrm{BD}}=\mathrm{MIC}_{1-\mathrm{BD}}+\mathrm{LEV} / \mathrm{MIC}_{1-\mathrm{BD}}$. Considering the $\mathrm{FICi}$ values, the nature of the interaction was as follow: $\leq 0.5$, synergy; $>0.5-1$, additive; $>1$ to $<2$, indifference; $\geq 2$ antagonism [23]. MIC values are geometric means of the replicate assays; the level of sample reproducibility was always within one-doubling-dilution difference that is satisfying in MIC testing.

\section{EtBr accumulation by fluorometric real-time efflux assay}

$\mathrm{EtBr}$, a fluorescent substrate of RND pumps that diffuses passively through the bacterial membrane and becomes strongly fluorescent after its irreversible binding to nucleic acids, has been used as a probe to evaluate the efflux activity of bacterial cells [24]. The assay was performed in a black flat bottom 96-well microplate, at equilibrium concentration of $\mathrm{EtBr}\left([\mathrm{EtBr}]_{\mathrm{eq}}\right)$, at which influx equals efflux [25]. To determine the [EtBr $]_{\mathrm{eq}}$, a cell suspension with $\mathrm{OD}_{600 \mathrm{~nm}}$ of 0.6 in a final volume of $150 \mu \mathrm{l}$ phosphate-buffered saline $(\mathrm{pH} 7.4$ ) with $0.4 \%$ glucose was exposed for $60 \mathrm{~min}$ at $37^{\circ} \mathrm{C}$, in the absence of $1-\mathrm{BD}$, at increasing concentration of $\mathrm{EtBr}$ (from 0.0625 to $6 \mu \mathrm{g} / \mathrm{ml}$ ). The choice of this range of $\mathrm{EtBr}$ concentrations was based on data reported in literature about the EtBr sensitivity of E. coli AG100 strains isogenic to our ones [25]. Concentrations of EtBr up to $5 \mu \mathrm{g} / \mathrm{ml}$ did not affect the growth of an E. coli AG100 mutant not expressing AcrAB while $300 \mu \mathrm{g} / \mathrm{ml}$ of EtBr are needed to inhibit the growth of a AG100 mutant overexpressing AcrAB [25]. The microplate was read at intervals of 60 sec with the Tecan infinite M200 PRO reader, excitation and detection wavelength of fluorescence were $530 \mathrm{~nm}$ and $585 \mathrm{~nm}$, respectively.

The effect of $1-\mathrm{BD}$ on the accumulation of $\mathrm{EtBr}$ was then evaluated at $[\mathrm{EtBr}]_{\mathrm{eq}}$, in $E$. coli cells treated with 0.8 (1/2 MIC) or $0.4 \mathrm{mg} / \mathrm{ml} 1-\mathrm{BD}(1 / 4 \mathrm{MIC})$; untreated cells were used as control. The accumulation of EtBr was reported as the relative final fluorescence $(R F F)$ index: $R F F=\left(R F_{\text {treated }}-R F_{\text {untreted }}\right) / R F_{\text {untreated }}$. An $R F F$ index $>1$ indicates an evident increased accumulation of EtBr compared with the control (non-treated cells taken as 0 ). All experiments were done in duplicate. A Student's $t$-test has been applied to evaluate statistically significant differences.

\section{Membranes permeability assay}

The effect of 1-BD on membranes integrity was evaluated using the Live/Dead BacLight Bacterial Viability Kit (Life Technology, MA, USA). E. coli strains were grown at $37^{\circ} \mathrm{C}$ with shaking until $\mathrm{OD}_{600 \mathrm{~nm}}$ of 0.12 . Aliquots of $0.5 \mathrm{ml}$ of $E$. coli cultures were incubated $1 \mathrm{~h}$ at $25^{\circ} \mathrm{C}$ with $1-\mathrm{BD}$ concentrations from 0.2 to $0.8 \mathrm{mg} / \mathrm{ml}$. Bacterial cells were centrifuged $\left(10,000 \times g\right.$ for $10 \mathrm{~min}$ a $\left.5^{\circ} \mathrm{C}\right)$ and resuspended in $0.5 \mathrm{ml}$ of a saline solution. $100 \mu \mathrm{l}$ of cell suspension were transferred into a black flat 96-well microtiter plate, where $50 \mu \mathrm{l}$ of the SYTO 9/propidium iodide dyes (ratio 1:1) were added to each well and the final volume adjusted to $200 \mu$ with distilled water. The microplate was incubated $15 \mathrm{~min}$ at room temperature in the dark. A Tecan infinite M200 PRO microplate reader set at fluorescence excitation wavelength of $485 \mathrm{~nm}$, was used to measure the fluorescence of SYTO 9 (green) and propidium iodide (red) at emission wavelength of $528 \mathrm{~nm}$ and $590 \mathrm{~nm}$, respectively. Bacteria with intact cell membranes fluoresced green and bacteria with compromised membranes fluoresced red. The green/red ratios were determined and the results were reported as the percentages of intact membranes in treated cells compared with the untreated control. All experiments were done in duplicate. A Student's $t$-test has been applied to evaluate statistically significant differences.

\section{Membrane potential assay}

The effect of 1-BD on membrane PMF was measured using the BacLight Bacterial Membrane Potential Kit (Life Technology). 1-BD was serially diluted $1: 2$ in sterile distilled water from 3.2 to $0.4 \mathrm{mg} / \mathrm{ml}$ into black flat bottom 96-well plates, and $5 \times 10^{5} \mathrm{cell} / \mathrm{ml} \mathrm{E}$. coli cells from fresh cultures and $30 \mu \mathrm{M}$ of the green fluorescent 3,3'-diethyloxacarbocyanine iodide were added to each sample to reach a final volume of $150 \mu$; the plates were incubated statically for $30 \mathrm{~min}$ at room temperature in the dark. Negative (water instead of 1-BD) and positive (78 $\mu \mathrm{M}$ carbonyl cyanide-m-chlorophenylhydrazone, to dissipate the PMF) controls were also present. 3,3'-Diethyloxacarbocyanine iodide fluoresces green, but the fluorescence shifts toward red when it is in a selfassociated form, as it happens when the cell depolarizes and its cytosolic concentration increases. A microplate reader Tecan infinite M200 PRO, set at excitation and detection wavelength of $528 \mathrm{~nm}$ (green) and $590 \mathrm{~nm}$ (red), respectively, was used to measure fluorescence. The red-green ratio was determined and expressed as percentage of depolarized membranes by normalizing against the emission from the negative control. All experiments were done in duplicate. A Student's $t$-test has been applied to evaluate statistically significant differences. 
Table 2. Adjuvant effect, of $1 / 4$ minimum inhibitory concentration of 1-benzyl-1,4-diazepane $(0.4 \mathrm{mg} / \mathrm{ml})$ and 1-(1-naphthylmethyl)piperazine $(0.1 \mathrm{mg} / \mathrm{ml})$, expressed as gain in antibiotic activity (A), in 1-DC14PS, 2-DC14PS and 3-AG100MKX Escherichia coli strains.

\begin{tabular}{|c|c|c|c|c|c|c|}
\hline \multirow[t]{2}{*}{ Strain } & \multirow[t]{2}{*}{ Antibiotic } & \multicolumn{3}{|c|}{$\operatorname{MIC}(\mu \mathrm{g} / \mathrm{ml})$} & \multicolumn{2}{|c|}{ Antibiotic activity gain (A) } \\
\hline & & No EPI & 1-BD & NMP & 1-BD & NMP \\
\hline \multirow[t]{4}{*}{ 1-DC14PS } & LEV & 0.25 & 0.25 & 0.25 & 1 & 1 \\
\hline & $\mathrm{CHL}$ & 2 & 2 & 2 & 1 & 1 \\
\hline & TET & 4 & 2 & 2 & 2 & 2 \\
\hline & RIF & 32 & 32 & 4 & 1 & 8 \\
\hline \multirow[t]{4}{*}{ 2-DC14PS } & LEV & 4 & 0.5 & 0.25 & 8 & 16 \\
\hline & $\mathrm{CHL}$ & 8 & 2 & 2 & 4 & 4 \\
\hline & TET & 8 & 4 & 2 & 2 & 4 \\
\hline & RIF & 8 & 32 & 8 & 0.25 & 1 \\
\hline \multirow[t]{4}{*}{ 3-AG100MKX } & LEV & 4 & 1 & 0.5 & 4 & 8 \\
\hline & $\mathrm{CHL}$ & 8 & 4 & 8 & 2 & 1 \\
\hline & TET & 8 & 4 & 4 & 2 & 2 \\
\hline & RIF & 32 & 32 & 8 & 1 & 4 \\
\hline
\end{tabular}

1-BD: 1-Benzyl-1,4-diazepane; CHL: Chloramphenicol; EPI: Efflux pump inhibitor; LEV: Levofloxacin; MIC: Minimum inhibitory concentration; NMP: 1-(1-Naphthylmethyl)piperazine; RIF: Rifampicin; TET: Tetracycline.

\section{Quantitative PALM experiments}

$1-\mathrm{BD}$ was characterized in its effect on the expression level of AcrB with quantitative super-resolution fluorescence microscopy. E. coli strain TV001 was grown at $37^{\circ} \mathrm{C}$ with shaking (210 r.p.m.) overnight. Afterward, the cells were diluted 1:100 in fresh LB medium and kept at $37^{\circ} \mathrm{C}$ with shaking in vented tubes for $2 \mathrm{~h}$ to reach the logarithmic growth phase. After $2 \mathrm{~h}$, bacteria were treated with $1-\mathrm{BD}$ at $0.4 \mathrm{mg} / \mathrm{ml}$ final concentration or sodium salicylate at $0.8 \mathrm{mg} / \mathrm{ml}$ final concentration. A third sample was left untreated as a control. Bacteria were exposed to treatments for $2 \mathrm{~h}$ while shaking at $37^{\circ} \mathrm{C}$. After two washing steps by centrifuging at $5000 \times g$ for 5 min at room temperature and resuspending in phosphate-buffered saline (PBS), cells were fixed by resuspending the pellet in $0.5 \mathrm{ml}$ of a $4 \%$ paraformaldehyde solution in PBS and incubating them at room temperature for $10 \mathrm{~min}$. After fixation, the samples were washed twice by centrifuging and resuspending the pellet in $1 \mathrm{ml}$ of PBS. The samples were stored at $4{ }^{\circ} \mathrm{C}$ for less than $24 \mathrm{~h}$ prior to imaging. Imaging chambers were built by placing microscope slides on top of poly-L-lysine-coated coverslips, separating them with thin strips of double sticky tape [26]. The bacterial suspensions were mounted for imaging by fluxing them within the chambers and letting them adhere to the poly-L-lysine-coated coverslips for at least $20 \mathrm{~min}$.

Imaging was performed on a custom-built widefield fluorescence microscope with single-molecule detection capabilities [26-28]. PALM was executed by activating PAmCherry with $405 \mathrm{~nm}$ light and recording the fluorescence signal emitted by single fluorescent proteins under $532 \mathrm{~nm}$ excitation until complete photobleaching of PAmCherry was observed. Acquired videos were analyzed with the ThunderSTORM ImageJ plugin [29] in order to reconstruct super-resolved molecular maps. Assignment of localized molecules to individual bacteria was then performed through a custom-made Matlab pipeline (Vignolini et al., Manuscript in Prep.). All experiments were done in triplicate. A Student's $t$-test has been applied to evaluate statistically significant differences.

\section{Results}

Antimicrobial activity of 1-BD, NMP \& antibiotics in E. coli strains overproducing or lacking major EPs of the RND type

MICs of 1-BD and NMP against different E. coli strains are reported in Supplementary Table 1. 1-BD was less toxic compared with NMP. $\mathrm{MIC}_{1-\mathrm{BD}}$ was $1.6 \mathrm{mg} / \mathrm{ml}$ in both pump-deficient and pump-overexpressing strains; similarly, $\mathrm{MIC}_{\mathrm{NMP}}$ was the same in all the tested strains, even though lower $(0.4 \mathrm{mg} / \mathrm{ml})$ than $\mathrm{MIC}_{1-\mathrm{BD}}$.

Table 2 reports the MICs of different antibiotics in the same E. coli strains. As expected, antibiotics that are substrates of AcrAB and AcrEF pumps, LEV, CHL and TET had MICs that were 16, 4 and 2, respectively, times higher in the overexpressing strains 2-DC14PS and 3-AG100MKX compared with 1-DC14PS strain. Whereas MIC values of RIF, an antibiotic that is considered a rather poor substrate of $E$. coli RND type EPs [30], were higher 
Table 3. Accumulation of ethidium bromide in Escherichia coli, expressed as relative final fluorescence (RFF).

\begin{tabular}{|c|c|c|c|}
\hline \multirow[t]{2}{*}{ Strain } & \multirow[t]{2}{*}{ 1-BD $(\mathrm{mg} / \mathrm{ml})$} & \multicolumn{2}{|c|}{$\mathrm{EtBr}(\mu \mathrm{g} / \mathrm{ml})$} \\
\hline & & 6 & 0.5 \\
\hline \multirow[t]{2}{*}{ DC14PS } & 0.8 & - & $0.70 \pm 0.56$ \\
\hline & 0.4 & - & $0.33 \pm 0.10^{\dagger}$ \\
\hline \multirow[t]{2}{*}{ 1-DC14PS } & 0.8 & - & $1.07 \pm 0.61$ \\
\hline & 0.4 & - & $0.60 \pm 0.01$ \\
\hline \multirow[t]{2}{*}{ 3-AG100MKX } & 0.8 & $3.66 \pm 2.18$ & - \\
\hline & 0.4 & $2.00 \pm 0.50^{\dagger}$ & - \\
\hline \multirow[t]{2}{*}{ 2-DC14PS } & 0.8 & $3.92 \pm 0.03$ & - \\
\hline & 0.4 & $2.49 \pm 1.34$ & - \\
\hline \multicolumn{4}{|c|}{$\begin{array}{l}\text { Note: RFF values at [EtBr]eq in E. coli strains DC14PS and 1-DC14PS }(0.5 \mu \mathrm{g} / \mathrm{ml} \text { of EtBr), 3-AG100MKX and 2-DC14PS }(6 \mu \mathrm{g} / \mathrm{ml}) \text { in the presence of subtoxic concentrations of 1-benzy } \\
\text { 1,4-diazepane. The data represent mean values } \pm \text { standard deviation from two independent experiments. } \\
+ \text { RFF values with statistically significant difference }(\mathrm{p}<0.05) \text { according to Student's } t \text {-test. } \\
\text { 1-BD: 1-Benzyl-1,4-diazepane; EtBr: Ethidium bromide; [EtBr]eq: Equilibrium concentration of EtBr; RFF: Relative final fluorescence. }\end{array}$} \\
\hline
\end{tabular}

and similar in 1-DC14PS and 3-AG100MKX, respectively, but four times lower in 2-DC14PS, suggesting that RIF is anyway a better substrate for AcrAB than for AcrEF.

\section{1-BD potentiates the antibacterial activity of different antibiotic against $E$. coli}

As reported in Table 2 , in the presence of $0.4 \mathrm{mg} / \mathrm{ml} 1-\mathrm{BD}(1 / 4 \mathrm{MIC})$, a potentiated activity $(\mathrm{A}>1)$ has been highlighted in 2-DC14PS and 3-AG100MKX for LEV ( $\mathrm{A}=8$ and $\mathrm{A}=4$, respectively) and CHL $(\mathrm{A}=4$ and $\mathrm{A}=2$ ), but not in 1-DC14PS (A = 1 for both LEV and CHL), whereas for TET a potentiated effect $(A=2)$ was detected in all strains. As expected for an antibiotic that is a poor substrate of RND pumps, 1-BD does not enhance the effect of RIF $(A \leq 1)$ in any strain, rather it appears to depower RIF in 2-DC14PS $(A=0.25)$, by what mechanism it is not easy to imagine. The reference EPI NMP at $1 / 4 \mathrm{MIC}(0.1 \mathrm{mg} / \mathrm{ml})$ acted similarly but, in contradiction with previous results [16,30], it did not enhance the activity of CHL $(A=1)$ in 3-AG100MKX.

The effect of 1-BD on LEV activity from two independent checkerboard assays, whose results were perfectly matching, are reported in Supplementary Table 2. In 3-AG100MKX and 2-DC14PS, overexpressing AcrAB and AcrEF, respectively, $0.4 \mathrm{mg} / \mathrm{ml}$ of $1-\mathrm{BD}$ determined a synergic effect $(\mathrm{FICi} \leq 0.5)$ on $\mathrm{LEV}$, whereas at lower concentration 1-BD showed an additive effect (FICi $>0.5-1)$. In any case, indifference (FICi $>1)$ characterized the 1-BD/LEV interaction in the 1-DC14PS strain lacking AcrAB.

\section{1-BD inhibits the efflux of EtBr}

The gain in antibiotic activity (A) and FICi consider bacterial growth inhibition to evaluate 1-BD effect on antibiotic efflux. A more direct measure of EPI activity of 1-BD was obtained by measuring the intracellular accumulation of EtBr by real-time fluorometric assay.

The accumulation kinetics of $\mathrm{EtBr}$ in the absence of 1-BD, in order to determine the $[\mathrm{EtBr}]_{\mathrm{eq}}$ in each E. coli strain, is reported in Supplementary Figure 1. As expected for a pump substrate, $[\mathrm{EtBr}]_{\mathrm{eq}}$ was found to be dependent on the EP expression. The AcrAB and AcrEF overexpressing mutants, 3-AG100MKX and 2-DC14PS respectively, had a $[\mathrm{EtBr}]_{\mathrm{eq}}$ higher $(6 \mu \mathrm{g} / \mathrm{ml})$ than the AcrAB-deficient mutants DC14PS and 1-DC14PS $(0.5 \mu \mathrm{g} / \mathrm{ml})$.

The addition of $1-\mathrm{BD}$ at $[\mathrm{EtBr}]_{\mathrm{eq}}$ resulted in a concentration-dependent increase in relative fluorescence intensity in both AcrAB-deficient and AcrAB-overexpressing strains (Figure 2). However, while in the deficient ones the increase was very low, in the overexpressing ones the $\mathrm{EtBr}$ accumulation was much higher, immediately reaching high levels. A quantitative evaluation of the ability of $1-\mathrm{BD}$ to promote the intracellular accumulation of EtBr is reported in Table 3 as RFF data, where relative is referred to the control without 1-BD. The EtBr accumulation results were always higher at $1-\mathrm{BD}$ concentration of $0.8 \mathrm{mg} / \mathrm{ml}$ than at $0.4 \mathrm{mg} / \mathrm{ml}$. Compared with controls without molecule, $1-\mathrm{BD}$ at $0.4 \mathrm{mg} / \mathrm{ml}(1 / 4 \mathrm{MIC})$, determines intracellular accumulation of EtBr in the EP overexpressing mutants (RFF $>1$ ), but not in the acr $A B$-deleted mutants (RFF $<1$ ). Similar results were also reported for NMP [16]. A significant difference was observed only between 1-DC14PS and 3-AG100MKX at $0.4 \mathrm{mg} / \mathrm{ml} 1$-BD. However, if the RFF values at $0.4 \mathrm{mg} / \mathrm{ml} 1-\mathrm{BD}$ of the two overexpressing strains were aggregated and the mean compared with the mean of the corresponding aggregated values of the AcrAB-deficient strains, a significant difference $(p<0.01)$ was observed. 

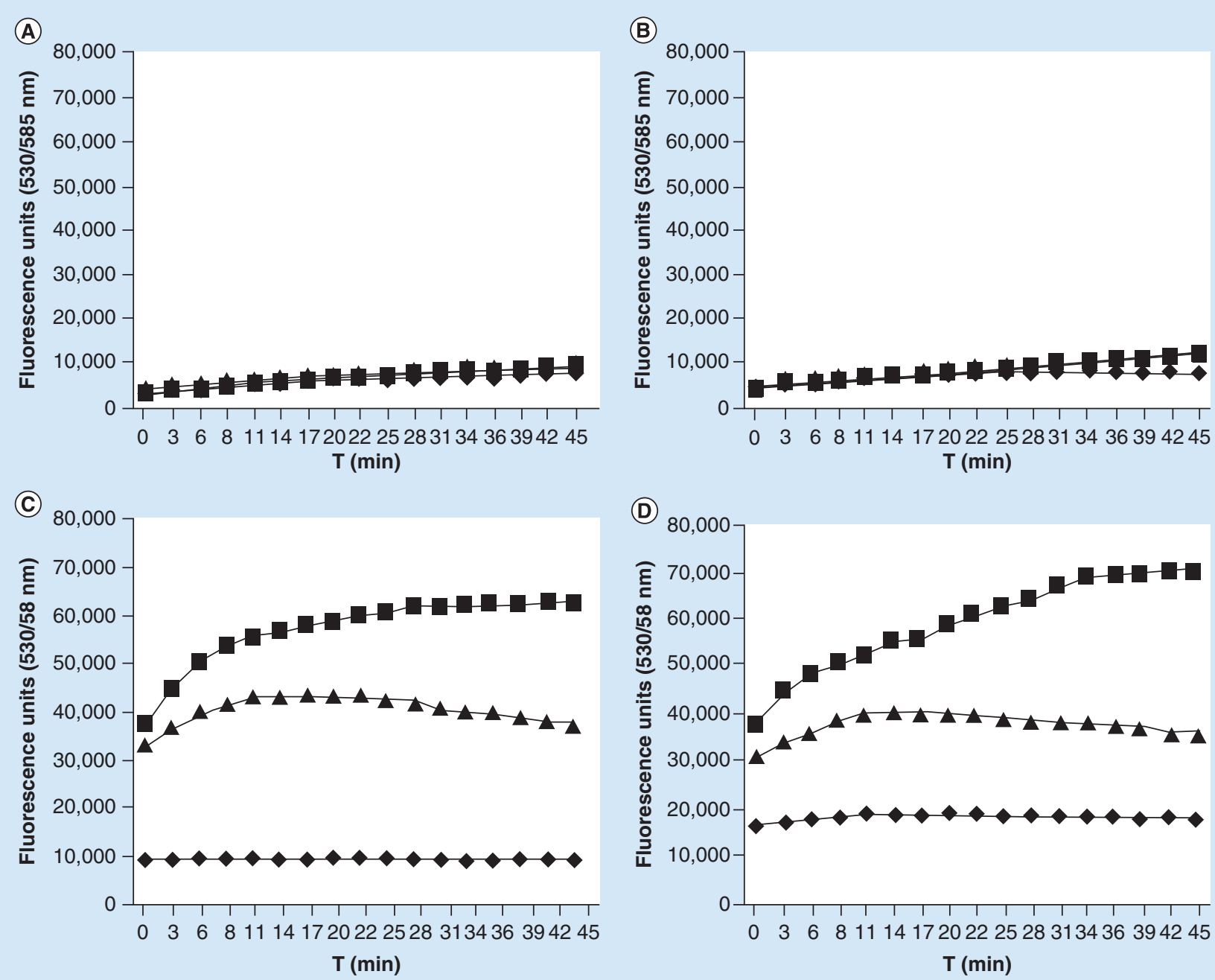

Figure 2. Effect of 1-BD on the accumulation of EtBr in Escherichia coli. (A) DC14PS; (B) 1-DC14PS; (C) 2-DC14PS and (D) 3-AG100MKX strains in the presence of [EtBr] eq $(0.5 \mu \mathrm{g} / \mathrm{ml}$ in $\mathbf{A}$ and $\mathbf{B} ; 6 \mu \mathrm{g} / \mathrm{ml}$ in $\mathbf{C}$ and $\mathbf{D})$ and at different concentration of 1-BD ( $\boldsymbol{\nabla}$, no molecule; $\mathbf{\Delta}$, $0.4 \mathrm{mg} / \mathrm{ml} ; \mathbf{\square}, 0.8 \mathrm{mg} / \mathrm{ml}$ ) at $37^{\circ} \mathrm{C}$ in the presence of glucose.

1-BD: 1-Benzyl-1,4-diazepane; EtBr: Ethidium bromide; [EtBr]eq: Equilibrium concentration of EtBr.

\section{1-BD affects the integrity of the membranes}

In Figure 3, the relative fluorescence green/red ratios of treated cells respect to untreated cells (green/red $=100 \%$ ) are reported. 1-BD induced a concentration dependent decrease of the integrity of the membranes (decrease in green/red ratio) in the three strains tested. $1-\mathrm{BD}$ at $0.4 \mathrm{mg} / \mathrm{ml}(1 / 4 \mathrm{MIC})$ caused integrity reduction of about 25 and $33 \%$ in the pump overexpressing mutants 3-AG100MKX and 2-DC14PS, respectively, significantly lower than that measured in 1-DC14PS (about $57 \%$ ). The permeabilization effect increased at the increase of $1-\mathrm{BD}$ concentration and the differences between the overexpressing strain and the AcrAB-deleted strains remained significant.

In all tested strains a modest IM depolarization effect by $1-\mathrm{BD}$ was observed, even at the highest concentrations of the molecule (Figure 4). Surprisingly, at $1 / 4 \mathrm{MIC}_{1-\mathrm{BD}}$, the depolarization in 3-AG100MKX (about 14\%) was significantly higher than that measured in 1-DC14PS and 2-DC14PS (about $2 \%$ in both strains). No significant differences were observed at higher concentration of 1-BD.

\section{1-BD decrease the expression of AcrB}

To investigate a possible effect of 1-BD on the expression level of $\mathrm{AcrB}$, we conducted quantitative single-molecule localization microscopy experiments on E. coli strain TV001, expressing a fluorescently-labeled version of AcrB 


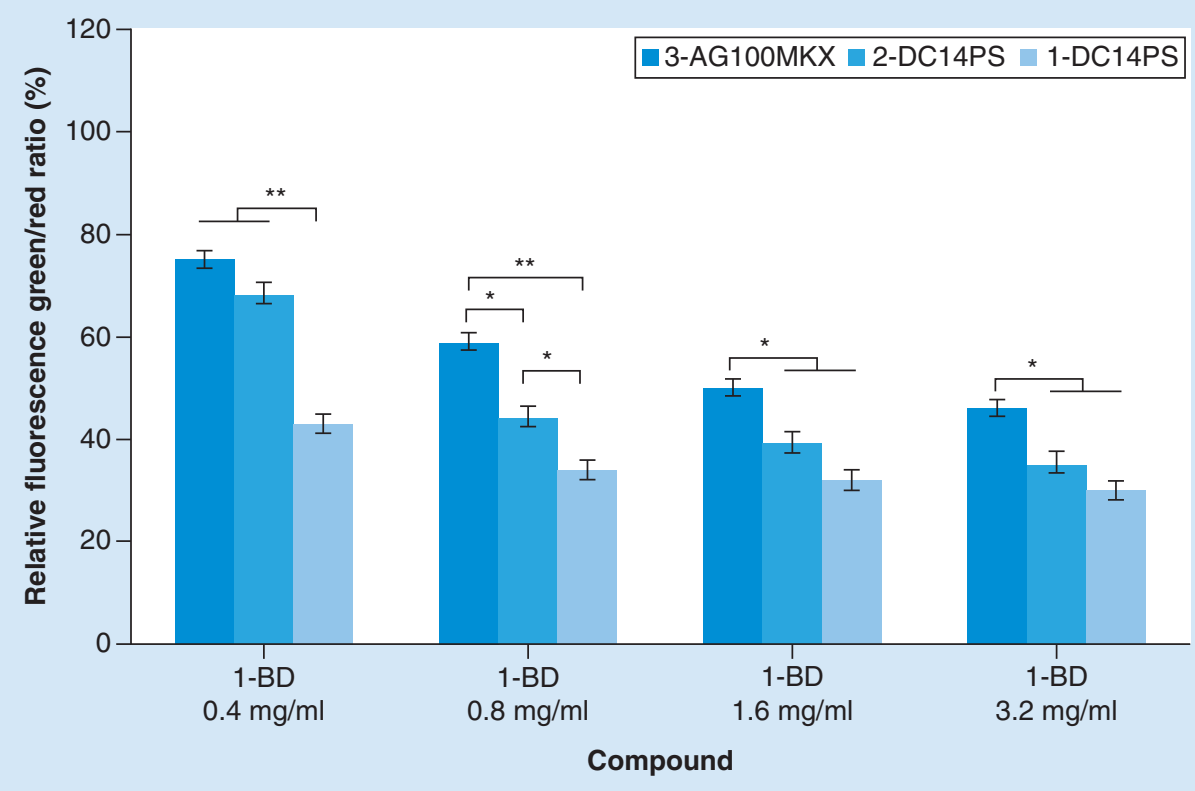

Figure 3. Effect of different concentrations of 1-benzyl-1,4-diazepane on the permeabilization of cell membranes in the Escherichia coli strains 3-AG100MKX, 2-DC14PS and 1-DC14PS. The values shown represent averages of two independent experiments and respective standard deviations. Asterisks indicate statistically significant differences according to Student's $t$-test. ${ }^{*} \mathrm{p}<0.05 ;{ }^{* *} \mathrm{p}<0.01$.

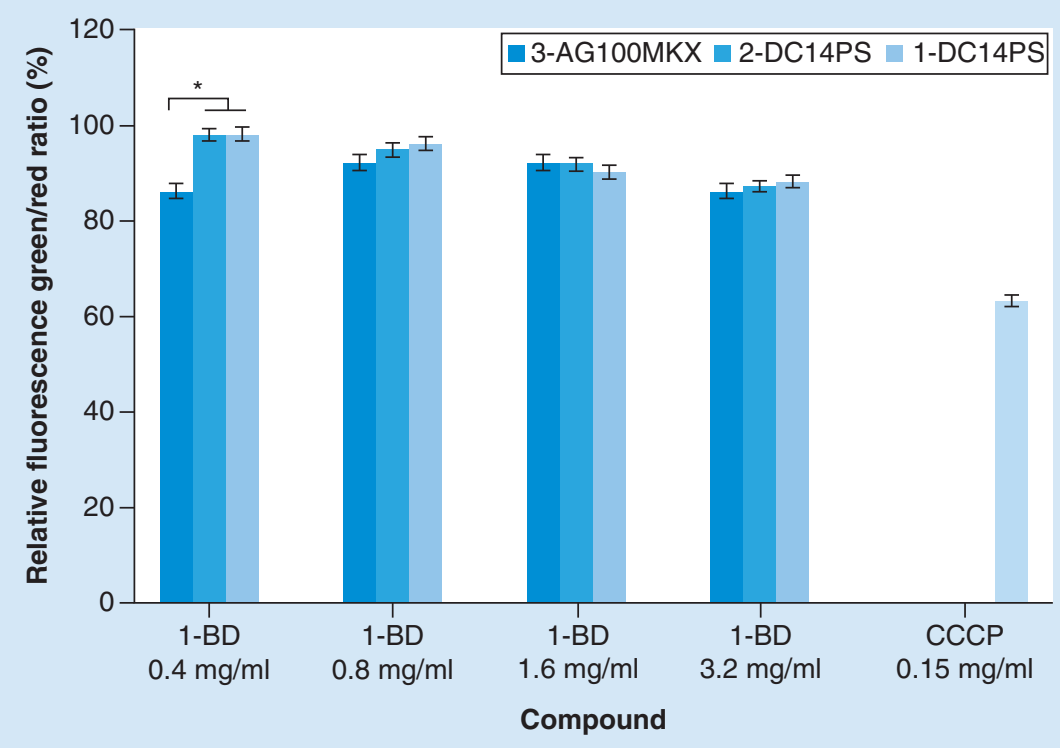

Figure 4. Effect of different concentrations of 1-benzyl-1,4-diazepane on inner membrane potential in the Escherichia coli strains 3-AG100MKX, 2-DC14PS and 1-DC14PS. The values shown represent averages of two independent experiments and respective standard deviations. CCCP was used as positive control for its ability to dissipate the proton gradient. Asterisks indicate statistically significant differences according to Student's $t$-test. ${ }^{*} \mathrm{p}<0.05$.

CCCP: Cyanide-m-chlorophenylhydrazone. 


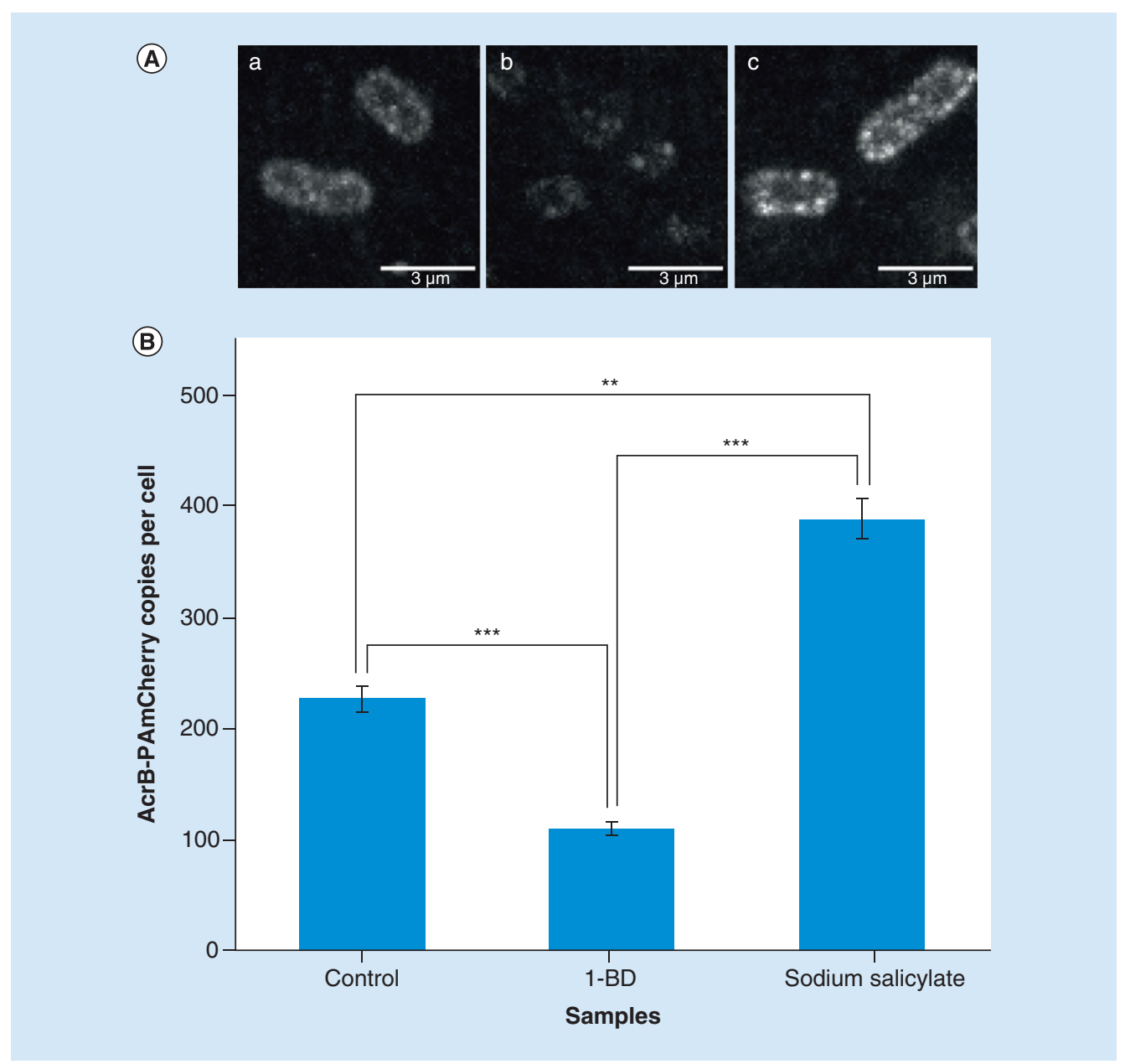

Figure 5. Effect of 1-benzyl-1,4-diazepane on the expression of AcrB in the Escherichia coli strain TV001 expressing AcrB fused to PAmCherry photoactivable fluorescent protein. (A) PALM images of E. coli TV001 cells after $2 \mathrm{~h}$ of incubation with either (a) plain LB medium (control), (b) $0.4 \mathrm{mg} / \mathrm{ml} \mathrm{1-BD} \mathrm{or} \mathrm{(c)} 0.8 \mathrm{mg} / \mathrm{ml}$ sodium salicylate. Each image is the result of a maximum intensity projection over 1000 consecutive frames. (B) Average number of AcrB-PAmCherry copies per bacterium after 2 h of incubation in control cells $(n=169)$, with 1-BD $(n=185)$ and sodium salicylate $(n=136)$ as a positive control. The values shown represent averages of three independent experiments and respective standard errors and were compared through Student's $t$-test ${ }^{*} p<0.05 ;{ }^{* *} p<0.01 ;{ }^{* *} p<0.001$.

1-BD: 1-Benzyl-1,4-diazepane; LB: Luria Bertani; PALM: Photoactivated localization microscopy.

from the original chromosomal locus. This technique allows for a direct measurement of the quantity of AcrB pump on the membrane of live bacteria under different conditions. In Figure 5, we report examples of the resulting fluorescence microscopy images of individual bacterial cells after $2 \mathrm{~h}$ of incubation with either 1-BD, sodium salicylate, or nothing (control). Sodium salicylate was used as a positive control for its ability to upregulate the expression of the $a c r A B$ operon by disrupting MarR-mediated repression [13,14]. Figure 5 also reports the average number of AcrB-PAmCherry copies observed by image analysis. There is a statistically significant decrease in the amount of observable AcrB-PAmCherry in bacterial cells after exposure to 1-BD ( $\mathrm{n}=185$ ) with respect to unexposed controls $(\mathrm{n}=169)$, which suggests an inhibitory effect of 1-BD on the expression of acrAB. The number of AcrB-PAmCherry copies in bacteria treated with 1-BD $(110.1 \pm 6.1)$ is about half of the copies measured in control cells $(226.5 \pm 11.2)$. On contrary, in the presence of sodium salicylate $(\mathrm{n}=136)$, AcrB-PAmCherry expression is roughly doubled (389.2 \pm 18.3$)$.

\section{Discussion}

The lack of effective treatment against infections caused by MDR bacteria is a worldwide healthcare emergency. Overexpression of EPs extruding antibiotics is a major mechanism in the resistance of clinical isolates [2]. For their 
essential role in removing antibiotics from bacterial cells increasing antibiotic resistance, EPs are very interesting target of combined, EPI plus antibiotic, therapies; an approach aimed to restore the effectiveness of the antibiotic in MDR bacterial pathogens. Developing clinically useful EPIs is a challenge; none of the so far identified EPIs have reached the clinical stage. This study reports the characterization of a novel EPI, 1-BD, able to inhibit major RND-type EPs of E. coli.

The ability of 1-BD, at subtoxic concentrations, to enhance the activity of LEV and other unrelated antibiotics (Table 2 \& Supplementary Table 2) and to decrease EtBr extrusion in the 3-AG100MKX and 2-DC14PS E. coli strains overexpressing AcrAB and AcrEF, respectively, but not in the DC14PS and 1-DC14PS strains lacking AcrAB (Table 3), strongly indicates that 1-BD would act as an inhibitor of AcrAB and AcrEF efflux capacity in E. coli. However, since the MIC of 1-BD was not influenced by the level of expression of Acr AB and AcrEF (Supplementary Table 1), 1-BD looks like a poor substrate of AcrAB and AcrEF. Consequently, like NMP and D13-9001 [1,31] and unlike $P A \beta N$ [1], it seems very unlikely that the ability of 1-BD to enhance the growth inhibitory activity of antibiotics is the result of competition with the antibiotic transport process by these pumps. 1-BD, independently from the expression levels of AcrAB and AcrEF, did not show any adjuvant effect on RIF (Table 2), an antibiotic that is not a substrate of these pumps; this behavior differentiates 1-BD from NMP (Table 2), and PA $\beta N$ [16]. As so far discussed, the inhibiting effect of 1-BD on the efflux of LEV and $\mathrm{EtBr}$ in $E$. coli could be ascribable to a more or less direct and specific effect on AcrAB and AcrEF activity. Anyway, by the above reported data we could not have excluded that 1-BD can also act by affecting membranes functionality. And in fact, 1-BD determinates a noticeable, concentration-dependent, permeabilization effect on membranes in $E$. coli (Figure 3). The effect was significantly higher in the acrAB knockout strain 1-DC14PS than in the E. coli strains overexpressing AcrAB and AcrEF, suggesting that a larger number of RND pumps complexes can protect cellular membranes (IM mostly) from the destabilization effects of 1-BD. A membrane permeabilization effect was also reported for PA $\beta \mathrm{N}$ in $E$. coli $[32,33]$ and P. aeruginosa [31], and for NMP in Klebsiella pneumonia [34], whereas D13-9001 did not permeabilized the IM in E. coli [33]. PA $\beta \mathrm{N}$ also affected the OM permeability in Pseudomonas aeruginosa with potency similar to polymyxin B nonapeptide, a compound well known for its permeabilizing activity on both IM and OM [31]. The contribution of the permeabilizing effect, by increasing antibiotics diffusion across membranes, must be considered in the evaluation of the adjuvant activity of 1-BD. The integrity of the IM is also essential for the preservation of the membrane potential, which supply the energy necessary to the operation of RND and other families of pumps.

1-BD showed little depolarizing effect on IM (Figure 4), such that the contribution of this effect to the observed enhancement of LEV activity and the accumulation of EtBr should also be very limited. A membrane potential reduction has been observed in Klebsiella pneumoniae exposed to NMP [34] and membrane depolarization is also the main mechanism for efflux-inhibition activity by the putative EPI 2-phenylquinolone (PQQ4R) in E. coli [32]. Even PA $\beta N$, not usually considered a proton conductor [1], has been reported to possess depolarizing activity in $E$. coli [33] and in P. aeruginosa [31].

For all of the above said, 1-BD appears to act as an antibiotic's enhancer by a mixed mechanism, which combines a noncompetitive inhibition of antibiotic transport by RND-type EPs to a membrane permeabilization activity. Everything considered, this mechanism seems different from those shown by the major EPIs NMP, PA $\beta N$, D13-9001 and PQQ4R. In addition to this dual mechanism of action, no doubt 1-BD also downregulates acrB expression, halving its presence on the IM of $E$. coli (Figures 5). The downregulation effect of 1-BD on acr $A B$ could be underestimated, because partially masked by 1-BD effect on IM permeability. In fact, as observed in Pseudomonas fluorescens, IM permeabilization could be responsible of enhanced expression of RND-type EPs, in the effort to repair membrane damage by increasing fatty acid efflux [35]. We can only speculate on how 1-BD downregulates the expression acrAB. Since salicylate is able to detach MarR from the marbox, inducing MarA expression and consequently that of $a c r A B$ and tolC genes [36], in the opposite way, 1-BD could bind to MarR, stabilizing it on the marbox of operon Mar, repressing the expression of acr $A B$ and $t o l C$ genes. However, as marR is disrupted in 3-AG100MKX strain, 1-BD could act downstream the expression cascade of acrAB. MarA or their homologues SoxS and Rob, involved in the transcription of marRAB and $A c r A B-T o l C$ [13] or AcrS/EnvR, known to represses the expression of $a c r A B$ and $a c r E F$, in $E$. coli [37], could be possible target candidates of 1-BD. As far as we know, none of the best characterized putative EPIs (NMP, PA $\beta N$, MBX2391 and D13-9001) have been reported to act by inhibiting the expression of $a c r A B$ or other EP genes in bacteria. However, different molecules from plant extracts have been reported, by transcription analysis or by reporter genes, to downregulate the expression of EP genes of the ABC class [10] and of AcrAB-TolC in E. coli [38] and Staphylococcus aureus [39]. Diclorofenac, a nonsteroidal anti-inflammatory drug, also strongly downregulated antimicrobial EPs of $S$. aureus [40]. In this work 
we used, for the first time, quantitative single-molecule localization microscopy to evaluate the effect of an EPI on the expression of an EP. This technique allows to look directly at the end product of the gene expression cascade, the AcrB protein, right where it is expected to be, in the IM.

$1-\mathrm{BD}$ showed a low cytotoxicity (in the mM range) in a preliminary MTS test on cultured primary Human Dermal Fibroblasts (data not shown). This result is encouraging because cytotoxicity is one of the major drawbacks to the clinical use of putative EPIs. For example, NMP and PA $\beta N$ are toxic because of their serotonin agonist properties [41] and membrane depolarizing activity [42], respectively.

\section{Conclusion}

1-BD shows most of the ideal characteristics of an EPI: it has no relevant intrinsic antibacterial activity (high MIC values); at subtoxic concentrations $(\leq 1 / 4 \mathrm{MIC})$, it enhances the activity of the antibiotics LEV, CHL, TET, substrates of EPs (but not that of the non-substrate RIF) in strains overexpressing EPs (3-AG100MKX and 2-DC14PS), but not in strains lacking AcrAB (DC14PS and 1-DC14PS); it decreases the extrusion of EtBr by AcrAB and AcrEF in 3-AG100MKX and 2-DC14PS, but not in 1-DC14PS; it has noticeable effects on membranes integrity but very little on proton gradient across the IM. Moreover, 1-BD downregulates acrB expression in $E$. coli. 1-BD seems to act by a mixed mechanism, overall different from that of so far quite well characterized EPIs. However, the knowledge of the large number of different EPs and the complex regulation of their expression in E. coli $[1,11]$ suggest caution in the interpretation of the data we report. Further investigation will be necessary for a more comprehensive view of the mechanism of action of 1-BD as EPI. Thanks to low cytotoxicity and good solubility in water, and despite its limited EPI activity, 1-BD is an attractive candidate as adjuvant to revitalize antibiotic therapy against resistant bacteria in a clinical setting. Structure-activity relationship studies will help to rationalize the design of 1-BD derivatives with enhanced and/or more specific activity.

\section{Summary points}

- 1-Benzyl-1,4-diazepane (1-BD) acts synergistically with levofloxacin and other antibiotics, enhancing their effects by two- to eightfold in Escherichia coli strains overexpressing efflux pumps AcrAB and AcrEF.

- 1-BD promotes the accumulation of ethidium bromide in the same E. coli strains.

- 1-BD increases membrane permeability, but only slightly affects membrane potential of $E$. coli cells.

- 1-BD halved the expression of acrB, as evaluated by quantitative photoactivated localization microscopy.

- The mechanism of action of 1-BD appears to be of a mixed type and overall different from that of major reference efflux pump inhibitors.

\section{Supplementary data}

To view the supplementary data that accompany this paper please visit the journal website at: www.futuremedicine.com/doi/sup $\mathrm{pl} / 10.2217 / \mathrm{fmb}-2019-0296$

\section{Acknowledgments}

We thank WV Kern for providing us with the E. coli strains DC14PS, 1-DC14PS, 2-DC14PS and 3-AG100MKX and L Piddock for providing us with the $E$. coli strain BW25113. The authors would like to thank F Bresci for technical support.

\section{Financial \& competing interests disclosure}

This work was supported by Fondazione CR Firenze (grant numbers 2016.1086, 2017.0750, 2017.0827); the European Union's Horizon 2020 research and innovation program grant no. 654148 Laserlab-Europe and by EMPIR project MetVBadBugs 15 HLT01. The authors have no other relevant affiliations or financial involvement with any organization or entity with a financial interest in or financial conflict with the subject matter or materials discussed in the manuscript apart from those disclosed.

No writing assistance was utilized in the production of this manuscript.

\section{References}

1. Li XZ, Plésiat P, Nikaido H. The challenge of efflux-mediated antibiotic resistance in Gram-negative bacteria. Clin. Microbiol. Rev. 28(2), 337-418 (2015).

2. Nikaido H, Pagès JM. Broad-specificity efflux pumps and their role in multidrug resistance of Gram-negative bacteria. FEMS Microbiol. Rev. 36(2), 340-363 (2012). 
3. Hernando-Amado S, Blanco P, Alcalde-Rico $\mathrm{M}$ et al. Multidrug efflux pumps as main players in intrinsic and acquired resistance to antimicrobials. Drug Resist. Update. 28, 13-27 (2016).

4. Krishnamoorthy G, Leus IV, Weeks JW et al. Synergy between active efflux and outer membrane diffusion defines rules of antibiotic permeation into Gram-negative bacteria. mBio 8(5), e01172-17 (2017).

5. Okusu H, Ma D, Nikaido H. AcrAB efflux pump plays a major role in the antibiotic resistance phenotype of Escherichia coli multiple-antibiotic-resistance (Mar) mutants. J. Bacteriol. 178(1), 306-308 (1996).

6. Du D, Wang Z, James NR et al. Structure of the AcrAB-TolC multidrug efflux pump. Nature 509(7501), 512-515 (2014).

7. Tikhonova EB, Zgurskaya HI. AcrA, AcrB, and TolC of Escherichia coli form a stable intermembrane multidrug efflux complex. J. Biol. Chem. 279(31), 32116-32124 (2004).

8. Sulavik MC, Houseweart C, Cramer C et al. Antibiotic susceptibility profiles of Escherichia coli strains lacking multidrug efflux pump genes. Antimicrob. Agents Chemother. 45(4), 1126-1136 (2001).

9. Anes J, McCusker MP, Fanning S et al. The ins and outs of RND efflux pumps in Escherichia coli. Front. Microbiol. 6, 587 (2015).

10. Spengler G, Kincses A, Gajdács $\mathrm{M}$ et al. New roads leading to old destinations: efflux pumps as targets to reverse multidrug resistance in bacteria. Molecules 22(3), e468 (2017).

11. Venter H, Mowla R, Ohene-Agyei T et al. RND-type drug efflux pumps from Gram-negative bacteria: molecular mechanism and inhibition. Front. Microbiol. 6, 377 (2015).

12. Durães F, Pinto M, Sousa E. Medicinal chemistry updates on bacterial efflux pump modulators. Curr. Med. Chem. 25(42), 6030-6069 (2018).

13. Weston N, Sharma P, Ricci V et al. Regulation of the AcrAB-TolC efflux pump in Enterobacteriaceae. Res. Microbiol. 169(7-8), 425-431 (2018).

14. Cohen SP, Levy SB, Foulds J et al. Salicylate induction of antibiotic resistance in Escherichia coli: activation of the mar operon and a mar-independent pathway. J. Bacteriol. 175(24), 7856-7862 (1993).

15. Tavío MM, Vila J, Perilli M et al. Enhanced active efflux, repression of porin synthesis and development of Mar phenotype by diazepam in two enterobacteria strains. J. Med. Microbiol. 53(Pt 11), 1119-1122 (2004).

16. Bohnert JA, Kern WV. Selected arylpiperazines are capable of reversing multidrug resistance in Escherichia coli overexpressing RND efflux pumps. Antimicrob. Agents Chemother. 49(2), 849-852 (2005).

17. Jellen-Ritter AS, Kern WV. Enhanced expression of the multidrug efflux pumps AcrAB and AcrEF associated with insertion element transposition in Escherichia coli mutants selected with a fluoroquinolone. Antimicrob. Agents Chemother. 45(5), 1467-1472 (2001).

18. Datsenko KA, Wanner BL. One-step inactivation of chromosomal genes in Escherichia coli K-12 using PCR products. Proc. Natl Acad. Sci. USA 97(12), 6640-6645 (2000).

19. Subach FV, Patterson GH, Manley S et al. Photoactivatable mCherry for high-resolution two-color fluorescence microscopy. Nat. Methods. 6(2), 153-159 (2009).

20. Reisch CR, Prather KL. The no-SCAR (Scarless Cas9 Assisted Recombineering) system for genome editing in Escherichia coli. Sci. Rep. 5 , 15096 (2015).

21. This reference is missing.

22. Clinical and Laboratory Standard Institute (CLSI). Methods for Dilutions Antimicrobial Susceptibility Tests for Bacteria That Growth Aerobically; Approved Standard (9th Edition) (M07-A9) CLSI, Clinical and Laboratory Standard Institute, PA, USA (2010). http://www.clsi.org

23. EUCAST - European Committee for Antimicrobial Susceptibility Testing. EUCAST Definitive Document E.Def 1.2, May 2000: terminology relating to methods for the determination of susceptibility of bacteria to antimicrobial agents. Clin. Microbiol. Infect. 6(9), 503-508 (2000).

24. Viveiros M, Martins A, Paixão L et al. Demonstration of intrinsic efflux activity of Escherichia coli K-12 AG100 by an automated ethidium bromide method. Int. J. Antimicrob. Agents 31(5), 458-462 (2008).

25. Paixão L, Rodrigues L, Couto I et al. Fluorometric determination of ethidium bromide efflux kinetics in Escherichia coli. J. Biol. Eng. 3, 18 (2009).

26. Gardini L, Calamai M, Hatakeyama $\mathrm{H}$ et al. Three-dimensional tracking of quantum dot-conjugated molecules in living cells. Method. Mol. Biol. 1814, 425-448 (2018).

27. Gardini L, Capitanio M, Pavone FS. 3D tracking of single nanoparticles and quantum dots in living cells by out-of-focus imaging with diffraction pattern recognition. Sci. Rep. 5, 16088 (2015).

28. Gardini L, Heissler SM, Arbore C et al. Dissecting myosin-5B mechanosensitivity and calcium regulation at the single molecule level. Nat. Commun. 9(1), 2844 (2018).

29. Ovesný M, Křížek P, Borkovec J et al. ThunderSTORM: a comprehensive ImageJ plug-in for PALM and STORM data analysis and super-resolution imaging. Bioinformatics 30(16), 2389-2390 (2014). 
30. Kern WV, Steinke P, Schumacher A et al. Effect of 1-(1-naphthylmethyl)-piperazine, a novel putative efflux pump inhibitor, on antimicrobial drug susceptibility in clinical isolates of Escherichia coli. J. Antimicrob. Chemother. 57(2), 339-343 (2006).

31. Lamers RP, Cavallari JF, Burrows LL. The efflux inhibitor phenylalanine-arginine beta-naphthylamide (PAßN) permeabilizes the outer membrane of Gram-negative bacteria. PLoS ONE 8(3), e60666 (2013).

32. Machado D, Fernandes L, Costa SS et al. Mode of action of the 2-phenylquinoline efflux inhibitor PQQ4R against Escherichia coli. PeerJ 5, e3168 (2017).

33. Matsumoto $\mathrm{Y}$, Hayama K, Sakakihara $\mathrm{S}$ et al. Evaluation of multidrug efflux pump inhibitors by a new method using microfluidic channels. PLoS ONE 6(4), e18547 (2011).

34. Anes J, Sivasankaran SK, Muthappa DM et al. Exposure to sub-inhibitory concentrations of the chemosensitizer 1-(1-naphthylmethyl)-piperazine creates membrane destabilization in multi-drug resistant Klebsiella pneumoniae. Front. Microbiol. 10, 92 (2019).

35. Adebusuyi AA, Foght JM. An alternative physiological role for the EmhABC efflux pump in Pseudomonas fluorescens cLP6a. BMC Microbiol. 11, 252 (2011)

36. Sulavik MC, Gambino LF, Miller PF. The MarR repressor of the multiple antibiotic resistance (mar) operon in Escherichia coli: prototypic member of a family of bacterial regulatory proteins involved in sensing phenolic compounds. Mol. Med. 1, 436e46 (1995).

37. Hirakawa H, Takumi-Kobayashi A, Theisen U et al. AcrS/EnvR represses expression of the acrAB multidrug efflux genes in Escherichia coli. J. Bacteriol. 190(18), 6276-6279 (2008).

38. Zhou X, Jia F, Liu X et al. Total alkaloids of Sophorea alopecuroides-induced down-regulation of AcrAB-TolC efflux pump reverses susceptibility to ciprofloxacin in clinical multidrug resistant Escherichia coli isolates. Phytother. Res. 26(11), 1637-1643 (2012).

39. Salaheen S, Peng M, Joo J et al. Eradication and sensitization of methicillin resistant Staphylococcus aureus to methicillin with bioactive extracts of berry pomace. Front. Microbiol. 8, 253 (2017).

40. Riordan JT, Dupre JM, Cantore-Matyi SA et al. Alterations in the transcriptome and antibiotic susceptibility of Staphylococcus aureus grown in the presence of diclofenac. Ann. Clin. Microb. Antimicrob. 10, 30 (2011).

41. Pagès JM, Amaral L. Mechanisms of drug efflux and strategies to combat them: challenging the efflux pump of Gram-negative bacteria. Biochim. Biophys. Acta 1794(5), 826-833 (2009).

42. Fleeman RM, Debevec G, Antonen $\mathrm{K}$ et al. Identification of a novel polyamine scaffold with potent efflux pump inhibition activity toward multi-drug resistant bacterial pathogens. Front. Microbiol. 9, 1301 (2018). 
